\title{
HCV and Kidney Transplantation: Pushing the Limits for Living Donation Case Report and Mini Review
}

\author{
A. Rossetto ${ }^{1 *}$, G. Comai ${ }^{2}$, V. Cuna ${ }^{2}$, M.C. Morelli ${ }^{3}$, A. Siniscalchi ${ }^{4}$, V. Corradetti ${ }^{2}$, M. Del Gaudio ${ }^{1}$, C. Zanfi ${ }^{1}$, V. Bertuzzo ${ }^{1}$, L. Ma- \\ roni $^{1}$, F. Odaldi ${ }^{1}$, G. Frascaroli ${ }^{1}$, G. La Manna ${ }^{2}$, A. D'Errico ${ }^{5}$ and M. Ravaioli ${ }^{1}$
}

${ }^{1}$ Department of organ insufficiencies and transplantation, General Surgery and Transplantation, University Hospital of Bologna, Policlinico S.Orsola-Malpighi, Italy

${ }^{2}$ Department of organ insufficiencies and transplantation, Nephrology, Dialysis and Transplantation, University Hospital of Bologna, Policlinico S.Orsola-Malpighi, Italy

${ }^{3}$ Department of organ insufficiencies and transplantation, University Hospital of Bologna, Policlinico S.Orsola- Malpighi, Italy

${ }^{4}$ Department of organ insufficiencies and transplantation, Anesthesia and Intensive Care of Transplants, University Hospital of Bologna, Policlinico S.Orsola-Malpighi, Italy

${ }^{5}$ Department of Experimental, Diagnostic and Specialty Medicine, Pathology Unit, University Hospital of Bologna, Policlinico S.OrsolaMalpighi, Italy

"Corresponding author: Anna Rossetto, Department of organ insufficiencies and transplantation, General Surgery and Transplantation, University Hospital of Bologna, Policlinico S.Orsola-Malpighi, Italy. Email: anna.rossetto@aosp.bo.it

Citation: Rossetto A, Comai G, Cuna V, Morelli MC, Siniscalchi A, et al. (2019) HCV and Kidney Transplantation: Pushing the Limits for Living Donation Case Report and Mini Review. J Surg 12: 1229. DOI: 10.29011/2575-9760.001229

Received Date: 28 May, 2019; Accepted Date: 12 June, 2019; Published Date: 17 June, 2019

\section{Abstract}

Introduction: Kidney transplantation is the treatment of choice for end stage renal disease. Because organ shortage, strategies to expand the donor pool are continuously developing. Until recently, HCV infection and elderly were contraindications to living donation. Marginality criteria have been extended for age and for HCV positivity with the advent of new anti HCV drugs (DAA). We report a particular case of living donor kidney transplantation carried out at our center. Cases of this type are still very rare.

Clinical Case: This is the case of a kidney living donation from a donor (the father, 78 years old) previously treated with DAA for an acute HCV hepatitis (genotype 1b) with SVR to a recipient (the son, 52 years old) af-fected by IgA nephropathy on hemodialysis treatment. The transplant was uneventfully. The immunosuppres-sion was based on basiliximab, steroids, tacrolimus and mycophenolate. No indication for antiviral prophylaxis was given to the recipient but only weekly evaluation of HCV-RNA for 10 weeks after transplant (negative).

Discussion: DAA allows the eradication of the infection in almost all patients with remission of autoimmune manifestations and normalization of peripheral $\mathrm{T}$ and B-lymphocyte homeostasis. They could play a role in acute rejection by reactivating the homeostasis of the adaptive and innate immune response. Rarely, DAA cause nephrotoxicity. HCV positive donors represent an important economic saving: although HCV positivity decreases graft and patient survival, it represents a gain in terms of survival compared to prolonged hemodialy-sis, with DAA therapy. Living donation from old donors have shown good results, and the advantages of living donation compared to cadaveric donation seem 'to compensate' the marginality of graft in term of age. Living donation from HCV treated donor with SVR allows to avoid exposition to antiviral drugs and to the infection even if for a limited period of time (the time needed for antiviral therapy), without even developing anti-HCV antibodies.

Conclusions: Even if further studies are necessary and a longer follow up is needed, it is certainly promising to safely expand the pool of organs available, allowing an increasing number of patients to access to kidney transplant. 


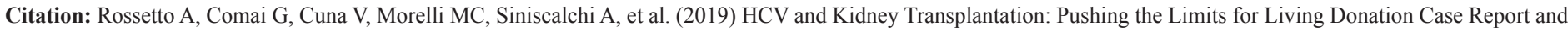
Mini Review. J Surg 12: 1229. DOI: 10.29011/2575-9760.001229

\section{Introduction}

End stage renal disease affects a large amount of people aroud the world. Even if kidney transplantation is not generally considered a 'life saving treatment', it is the treatment of choice for end stage renal disease, and it's worldwide accepted and demostrated by several sudies that it offers a better survival compared to dialysis, besides offering better quality of life and lower health care costs. Thus, people waiting in the waiting lists far exceeds the organ availability. Great attention is placed to continuously developing strategies trying to expand the organ pool using living donor programs extended in turn with the kidney paired donation, DCD program, and to maximize the use of available organs using marginal grafts (both from old patients or from increased risk donors) dual kidney transplantation, perfusion techniques to ameliorate quality reducing ischemia/perfusion injury. Also the living donor basin has been further expanded with the use of selected marginal donors (ie, who have no age parameters and ideal glomerular filtration rates or who have anemia or benign disease) demonstrating an acceptable outcome everywhere. The guidelines approved by the Japanese Transplantation Committee have considered usable as marginal living donors with ages between 71 and 80 years. From the infectious point of view, until very recently, HCV infection in the potential donor was considered a contraindication to living donation both for the high risk of transmitting $\mathrm{HCV}$ and for the risk of glomerular disease in the donor [1-4]

The advent of new anti HCV drugs era, with the introduction of direct-acting antiviral agents, has introduced a real revolution in the management of antiviral therapy for hepatitis C [5]. Given these assumptions, in view of the fact that a good proportion of organs is discarded as $\mathrm{HCV}$ positive and that many dialysis patients are affected by $\mathrm{HCV}$, many considerations with a view to optimizing the organ pool have been made. In particular, if the use of positive HCV organs on positive HCV recipient is already a reality $[6,7]$ studies have emerged on the possibility of transplanting positive $\mathrm{HCV}$ organs from cadaveric donor on $\mathrm{HCV}$ negative patients who undergo antiviral therapy after transplantation [6-11]. Consequently, the use of living donors can also be reviewed and expanded, considering donors who have obtained a sustained virological response. Cases of this type are still very rare $[10,12,13]$. The following is a case of a living donor kidney transplant carried out at our center; the donor was an old man who had been treated with the new drugs for a previous $\mathrm{HCV}$ infection and had obtained a SVR; the receiver was HCV negative.

\section{HCV and Kidney}

$\mathrm{HCV}$ infection can lead to renal damage in several ways; the most frequent pathways is the type I membranoproliferative glomerulonephritis associated with mixed cryoglobulinemia. Rarer forms include the membranoproliferative glomerulonephritis without cryoglobulinemia and membranous nephropathy.
However, there are other conditions that are associated with HCV infection and that are responsible for nephrotoxicity supported by systemic immunological responses to infection [7]. The kidney structure is also the perfect environment for the stages of the extra hepatic virus pathogenicity, expressing all the factors involved in the replication phases $[14,15]$.

A mechanism of virus entry into the kidney that would use infected B cells or endosomes as a Trojan horse was also hypothesized. Although the most common form of HCV-related renal damage due to cryoglobulinemia is likely that there are several direct effects of the same viral cell infection, through the interaction with membrane receptors that activate a cascade of cytopathic effects that result in endothelitis, mesangial inflammation and podocyte damage, which would justify the presence of renal damage even in the absence of cryoglobulinemia. Furthermore, the viral load also appears to correlate with the extent of renal damage [14]. In addition, HCV infection also has immunological significance for a number of reasons; on the one hand it seems to act as antigen significantly: the antibodies are able to form immune complexes with the rheumatoid factor in a preferential way compared to the other circulating $\operatorname{IgG}$; moreover, it is able to lower the activation threshold of $\mathrm{B}$ cell and $\mathrm{T}$ cells with an immune harmful effect to self $[7,14,16]$.

\section{Living Donation and Elderly}

Living kidney donation is a safe and well tolerated surgical procedure but not completely risk free [17]. In a fairly recent past, advanced age $(>60)$ was a contraindication to living donation. Today, living donors over the age of 60 constitute a good percentage of all living donors. In relation to the donor, in fact, we have seen that the substantial difference between $>60$ and $<60$ years would be attributable to factors related only to age and not to donation (higher incidence of hypertension and reduction of glomerular filtrate) [18]. In relation to the recipient, the age of the graft has weight on the outcome of the transplant. It has been shown that the renal function in graft from elderly living donors is lower than that of young adult donor graft, but there does not seem to be any substantial difference in terms of functionality decline and graft survival. In general, however, the living donor transplant, albeit marginal, gives results superior to those obtained from a cadaveric donor [19]. Considering the generic favorable aspects of living transplantation (reduced ischemia time, etc.), it would seem that the transplant from an elderly living donor could be comparable to a young cadaveric donor. What is actually the age threshold for considering donation is not really clear, by virtue of the requested combined evaluation between different characteristics of the donor (age, sex and glomerular filtrate). However, if the 60-year-old threshold can be said to have been exceeded, with extreme caution can be considered for living donation donors of more than 70 years of age [20]. 


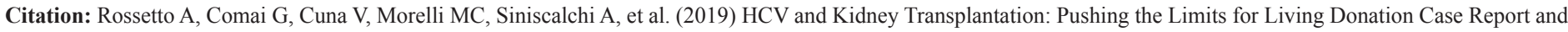
Mini Review. J Surg 12: 1229. DOI: 10.29011/2575-9760.001229

\section{Case Report}

We report a case of a negative anti-HCV kidney recipient from a donor (the father, 79 years old) previously treated with Elbasvir/Grazoprevir for an acute HCV hepatitis (genotype 1b) with sustained virological response 12 weeks after the end of therapy. The donor had in his medical history: arterial hypertension in drug therapy, benign prostatic hypertrophy in therapy; hypercholesterolemia, ex-tabagic habit (about 20 cigarettes / day for about 30 years), polydistrictal vasculopathy with calcifical parietal atheromas of the femoral-popliteal-tibial axes, appendectomy at a young age, left upper pulmonary lobectomy for adenocarcinoma in 1998 with negative follow-up, umbilical hernioplasty. On December 2017 he had been hospitalized for an episode of acute $\mathrm{HCV}$ related hepatitis (genotype $1 \mathrm{~b}$ ), following antiviral therapy with Elbasvir / Grazoprevir from June to September 2018 with sustained virological response, HCV-RNA negativization and persistence of HCV-RNA negativity 12 weeks after suspension of the therapy. Left nephrectomy was performed with left lombotomic access. The donor was discharged 7 days after the operation in good clinical conditions. The recipient was a man 52 years old (the son) affected by end stage renal disease (IgA nephropathy on hemodialysis treatment).

Blood group of the donor was 0 negative, the recipient was 0 positive. The cross-match was negative, antibodies research of anti-HLA class II was negative. The transplant was performed uneventfully, the kidney was normally placed in the left iliac fossa with packaging of ureter-bladder anastomosis and placement of ureteral stent to be removed at 5 weeks from the intervention. A renal biopsy was performed with no evidence of preservation damage, no reperfusion injury, no predictive damage of Primary Non Function (PNF) or Delayed Graft Function (DGF), absence of acute ischemic injury, absence of predictors, acute rejection, Karnoski score 4. Only the presence of diffuse, global, mesangial glomerular positivity was reported for $\mathrm{C} 4 \mathrm{~d}, 3+$, with negative $\mathrm{C} 3 \mathrm{~d}$, which seems to be able to be triggered in a simple way to simple phenomena of hyalinosis, even though it cannot exclude with absolute certainty other causes. The immunosuppressive therapy was based on Basiliximab, steroids, tacrolimus and mycophenolate. The recipient was discharged in 18 post-operative days. No indication for antiviral prophylaxis was given to the recipient given the negativization of $\mathrm{HCV}$ in the donor. He performed only weekly blood samples for the evaluation of HCV-RNA that were always negative after 10 weeks after transplant. The laboratory result for HCV antibodies was not interpretable because it was weakly positive initially and negative at a second review; therefore, we will proceed to the laboratory follow up of this parameter.

\section{Discussion}

Living donation is undoubtedly superior to cadaveric donation in terms of survival of the recipient and the graft due to the undeniable technical advantage deriving from the maximum reduction in ischemia times, the excellent condition of the donor and the programming of the intervention that makes it an elective intervention. The age, as presented before, does not seem to represent a contraindication since, even if cautiously, the rigid age limits of the past guidelines have been overcome. The ethical arguments on these topics are obviously strong. There are authors who in fact suggest that the risk of using an elderly living donor is actually much more predictable than the risk of using a young living donor, with a long life expectancy and with unknown and unpredictable possibilities of having complications related to donation [20]. Within the criteria to be followed in order to consider living donation, great attention is paid to the study of transmissible infections; according to the guidelines in fact, the infectious risk of the donor for HIV, HCV and HBV should be rigorously studied by repeating the serological tests within 28 days before the donation to minimize the risk of transmissible infection $[4,21,22]$.

In patients undergoing hemodialysis and kidney transplant patients the incidence of $\mathrm{HCV}$ is greater than in the general population; with great variability linked to the geographical areas it is around $10 \%$ in the US and Europe and most of patients are viremic [23]. Survival rate of hemodialys patients and kidney transplanted patients affects by $\mathrm{HCV}$ is lower compared to $\mathrm{HCV}$ negative patients [22]; the risk for sepsis, diabetes, glomerulonephritis is higher for these patients [15]. Higher rate of acute cellular rejection and higher prevalence of chronic allograft nephropathy have been reported in patients with long time antiHCV positivity [16,24]. Transplant recipients of kidney affected by HCV take 10-20 years to exhibit renal HCV related damage but there is instead few information on the possibility of developing kidney damage in patients who developed the infection in the period for transplantation [16]. The immunosuppressive drugs started after transplantation increases viral replication leading to pathological deterioration of liver in $75 \%$ of patients and liver cirrhosis in $25 \%$ of patients within 5 years [25]. The advent of new anti HCV drugs allows the eradication of the infection in almost all the treated patients offering also a complete remission of autoimmune manifestations as well as normalization of peripheral $\mathrm{T}$ and B-lymphocyte homeostasis [5], although it seems they are unable to restore the inflammatory-like constituent structure by expression of cytokines, chemokines, interferon-stimulated gene expression and in the Mucous-Associated Invariant T Cells (MAIT). These aspects would also be correlated with alterations in cytomegalovirus-specific and Epstein-Barr virus-specific T cells responses [26].

DAA are generally well tolerated. Few papers anyway report cases of renal damage DAA related such as acute immune complex glomerulonephritis, nephritic proteinuria, focal segmental glomerulosclerosis and late recurrence of membranous nephropathy $[9,21,27]$. Furthermore, it is interesting and not 


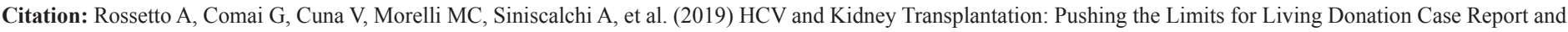
Mini Review. J Surg 12: 1229. DOI: 10.29011/2575-9760.001229

completely clarified the role that these drugs could play with regard to acute rejection if we exclude the interaction, that would seem minimal, with the immunosuppressive agents. It seems that they are able to interact with the immunological function by reactivating the homeostasis of the adaptive and innate immune response previously suppressed during chronic viral infection. In fact, the regression of the immunomediate phenomena correlated with HCV infection after eradication with DAA is reported. These phenomena, on the other hand, could be responsible for acute cellmediated rejection $[5,23]$.

Another interesting point is represented by seronegative occult hepatitis $\mathrm{C}$ virus infection: because of the characteristics of the virus there are hypotheses on the occult infection and works that highlight the persistence of the virus in the hepatocyte and in the mononuclear cells of the peripheral blood even for years by the spontaneous or pharmacological resolution of the HCV infection. Two type of occult infections have been reported: HCV RNA negative/Ab anti RNA negative or HCV RNA negative/Ab anti $\mathrm{HCV}$ positive. HCV RNA is present in liver of these patients and viral RNA can be detected in peripheral blood mononuclear cells [20]. However, there are also works that question the real substance of the problem [28-30]. There are studies that have demonstrated a clear saving in economic terms that may derive from the use of positive HCV donors; many patients may benefit from a shorter waiting time on the list and do not need prolonged hemodialysis treatments. In addition, the relative safety of new drugs could pave the way for pre-emptive treatments in the negative HCV recipients, further increasing the gain in waiting time. Although it seems clear that the HCV positivity decreases the graft and patient survival, no differences with respect to the antibody status alone in terms of graft and patient survival have been demonstrated at present $[31,32]$.

Even if further studies are necessary to confirm all these hypotheses and time to obtain a longer follow up is needed, it is certainly promising to be able to safely expand the pool of organs available, allowing an increasing number of patients to access the kidney transplant. If the timing of antiviral therapy is still not fully clarified for $\mathrm{HCV}$ population (before or after transplantation), probably a case like the one proposed by us could present various favorable conditions. In fact, in a situation like this the recipient has not been exposed to antiviral drugs, which although well tolerated, however, have a minimal nephrotoxicity and could interfere minimally with the immunosuppressive drugs and with the immunological response of the recipient in terms of development of acute rejection. Moreover, the recipient in this way would not be exposed to the infection even if for a limited period of time (the time needed for antiviral therapy), in addition benefiting from the undisputed advantages of living donation with a very limited period of hemodialysis and excellent results despite the advanced age of the donor.

\section{References}

1. Lee LY, Pham TA, Melcher ML (2019) Living Kidney Donation: Strategies to Increase the Donor Pool. Surg Clin North Am 99: 37-47.

2. Segall L, Nistor I, Pascual J, Mucsi I, Guirado L, et al. (2016) Criteria for and Appropriateness of Renal Transplantation in Elderly Patients with End-Stage Renal Disease: A Literature Review and Position Statement on Behalf of the European Renal Association-European Dialysis and Transplant Association Descartes Working Group and European Renal Best Practice. Transplantation 100: e55-65.

3. Habbous S, Woo J, Lam NN, Lentine KL, Cooper M, et al. (2018) The Efficiency of Evaluating Candidates for Living Kidney Donation: A Scoping Review. Transplant Direct 4: e394.

4. Lentine KL, Kasiske BL, Levey AS, Adams PL, Alberú J, et al. (2017) KDIGO Clinical Practice Guideline on the Evaluation and Care of Living Kidney Donors. Transplantation 101: S1-S109.

5. Comarmond C, Garrido M, Pol S, Desbois AC, Costopoulos M, et al. (2017) Direct-Acting Antiviral Therapy Restores Immune Tolerance to Patients with Hepatitis C Virus-Induced Cryoglobulinemia Vasculitis. Gastroenterology 152: 2052-2062.

6. Bhamidimarri KR, Ladino M, Pedraza F, Guerra G, Mattiazzi A, et al. (2017) Transplantation of kidneys from hepatitis C-positive donors into hepatitis $C$ virus-infected recipients followed by early initiation of direct acting antiviral therapy: a single-center retrospective study. Transpl Int 30: $865-873$

7. Joglekar K, Eason JD, Molnar MZ (2017) Do we really need more evidence to use hepatitis $C$ positive donor kidney more liberally? Clin Kidney J 10: 560-563.

8. Belga S, Doucette KE (2016) Hepatitis C in non-hepatic solid organ transplant candidates and recipients: A new horizon World J Gastroenterol 22: 1650-63.

9. Goldberg DS, Abt PL, Blumberg EA, Van Deerlin VM, Levine M, et al. (2017) Trial of Transplantation of HCV-Infected Kidneys into Uninfected Recipients.N Engl J Med 376: 2394-2395.

10. Agarwal N, Davis RJ, Gracey DM, Wong G, Kable K, et al. (2018) Detection of Hepatitis C Antibodies Without Viral Transmission in Hepatitis C-Negative Recipients Receiving Kidneys From Hepatitis CPositive Donors Treated With Direct-Acting Antiviral Therapy. Transplantation 102: e121-e122.

11. Durand CM, Bowring MG, Brown DM, Chattergoon MA, Massaccesi G, et al. (2018) Direct-Acting Antiviral Prophylaxis in Kidney Transplantation From Hepatitis C Virus-Infected Donors to Noninfected Recipients: An Open-Label Nonrandomized Trial. Ann Intern Med 168: 533-540.

12. Cruzado JM, Gil-Vernet S, Castellote J, Bestard O, Melilli E, et al. (2013) Successful treatment of chronic HCV infection should not preclude kidney donation to an HCV negative recipient. Am J Transplant 13: $2773-2774$

13. Gelpi R, Rodríguez-Villar C, Paredes D, Roque R, Ruiz A, et al. (2018) Safety of hepatitis $\mathrm{C}$ virus (HCV)-treated donors for kidney transplantation excluding occult HCV infection through kidney biopsies. Transpl Int 31: 938-939.

14. Minutolo R, Aghemo A, Chirianni A, Fabrizi F, Gesualdo L, et al. (2018) Management of hepatitis $C$ virus infection in patients with chronic kid- 


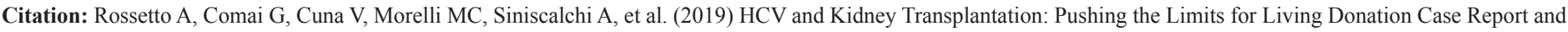
Mini Review. J Surg 12: 1229. DOI: 10.29011/2575-9760.001229

ney disease: position statement of the joint committee of Italian association for the study of the liver (AISF), Italian society of internal medicine (SIMI), Italian society of infectious and tropical disease (SIMIT) and Italian society of nephrology (SIN). Dig Liver Dis 50: 1133-1152.

15. Carrier P, Essig M, Debette-Gratien M, Sautereau D, Rousseau A, et al. (2016) Anti-hepatitis C virus drugs and kidney. World J Hepatol 8: 1343-1353.

16. Kesiraju S, Srikanti P, Sahariah S (2017) Hepatitis C infection in renal transplantation: pathogenesis, current impact and emerging trends. Virus disease 28: 233-241.

17. Ravaioli M, Capocasale E, Furian L, De Pace V, laria M, et al. (2017) Are there any relations among transplant centre volume, surgical technique and anatomy for donor graft selection? Ten-year multicentric Italian experience on mini-invasive living donor nephrectomy. Nephrol Dial Transplant 32: 2126-2131.

18. Serrano OK, Yadav K, Bangdiwala A, Vock DM, Dunn TB, et al. (2018) Age alone is not a contraindication to kidney donation: Outcomes of donor nephrectomy in the elderly. Clin Transplant 32: e13287.

19. Oikawa M, Hatakeyama S, Narita T, Yamamoto H, Hosogoe S, et al. (2016) Safety and Effectiveness of Marginal Donor in Living Kidney Transplantation. Transplant Proc 48: 701-705.

20. Hourmant M, Lerat L, Karam G (2013) Donation from old living donors: how safe is it? Nephrol Dial Transplant 28: 2010-2014.

21. Grossi PA, Dalla Gasperina D, Lombardi D, Ricci A, Piccolo G, et al. (2018) Organ transplantation from "increased infectious risk donors": the experience of the Nord Italia Transplant program- A retrospective study. Transpl Int 31: 212-219.

22. Irwin L, Kotton CN, Elias N, Palafox J, Basler D, et al. (2017) Utilization of increased risk for transmission of infectious disease donor organs in solid organ transplantation: Retrospective analysis of disease transmission and safety. Transpl Infect Dis 19.

23. Murakami N, Ding Y, Cohen DJ, Chandraker AK, Rennke HG (2018) Recurrent membranous nephropathy and acute cellular rejection in a patient treated with direct anti-HCV therapy (ledipasvir/sofosbuvir). Transpl Infect Dis 20.
24. Garimella T, Wang R, Luo WL, Hwang C, Sherman D, et al. (2015) Single-dose pharmacokinetics and safety of daclatasvir in subjects with renal function impairment. Antivir Ther 20: 535-543.

25. Mahmoud IM, Sobh MA, El-Habashi AF, Sally ST, El-Baz M, et al. (2005) Interferon therapy in hemodialysis patients with chronic hepatitis C: study of tolerance, efficacy and post-transplantation course. Nephron Clin Pract 100: c133-139.

26. Hengst J, Falk CS, Schlaphoff V, Deterding K, Manns MP, et al. (2016) Direct-Acting Antiviral-Induced Hepatitis C Virus Clearance Does Not Completely Restore the Altered Cytokine and Chemokine Milieu in Patients with Chronic Hepatitis C. Infect Dis 214: 1965-1974.

27. Hogan JJ, Lim MA, Palmer MB, Bloom RD, Chung RT, et al. (2017) Development of proteinuria and focal segmental glomerulosclerosis during direct-acting antiviral therapy for hepatitis $\mathrm{C}$ virus infection. $\mathrm{He}-$ patology 66: 658-660.

28. Castillo I, Martinez-Ara J, Olea T, Bartolomé J, Madero R, et al. (2014) High prevalence of occult hepatitis $C$ virus infection in patients with primary and secondary glomerular nephropathies. Kidney Int 86: 619624.

29. Yamabe H, Nakamura N, Nakamura M, Shimada M, Kumasaka R, et al. (2005) Hepatitis C virus-associated glomerulonephritis without hepatitis $C$ virus in the blood. Am J Kidney Dis 46: e65-69.

30. Pham TN, MacParland SA, Mulrooney PM, Cooksley H, Naoumov NV, et al. (2004) Hepatitis $C$ virus persistence after spontaneous or treatment-induced resolution of hepatitis C. J Virol 78: 5867-5874.

31. Gupta G, Zhang Y, Carroll NV, Sterling RK (2018) Cost-effectiveness of hepatitis C-positive donor kidney transplantation for hepatitis C-negative recipients with concomitant direct-acting antiviral therapy. Am J Transplant 18: 2496-2505

32. Maluf DG, Archer KJ, Mas VR (2010) Kidney grafts from HCV-positive donors: advantages and disadvantages. Transplant Proc 42: 24362446 . 